\title{
Analysis of Big Data Cloud Storage Technology Based on Information Fusion Technology
}

\author{
Shengbin Wu \\ Changsha Medical University, Changsha, Hunan, 410219, China
}

Keywords: Storage technology, Information fusion technology, Big data

\begin{abstract}
Compared with traditional storage technology, cloud storage technology is more advanced, mainly relying on server clusters to manage related information on the basis of network software coordination. This technology has strong applicability and convenient operation, which greatly improves the quality of people's work. However, due to the openness of this technology, all its security issues are more prominent. For this reason, the author mainly discusses the cloud storage technology under the big data scenario, and focuses on the analysis and exploration of its specific applications, in order to help people make full use of the technology.
\end{abstract}

\section{Introduction}

Fusion in information fusion technology refers to the collection and integration of multiple information sources, multimedia and multi-format information to form effective comprehensive information in a timely, accurate and complete manner. Its concept first appeared in the early 1970s. After combining multi-sensor data and auxiliary databases, the inference results obtained by data fusion technology are more accurate than a single sensor. Multi-sensor information has the characteristics of redundancy, coordination, compensation, low cost and real-time after fusion. Information fusion technology is a synthesis of multiple traditional disciplines such as digital statistical estimation, signal processing, control theory, classical digital methods, and artificial intelligence, with algorithms related to information fusion as the main content of the research. Multi-sensor information fusion technology is mainly used in signal detection, tracking, robot navigation, image fusion and other fields, and its application prospects in transportation, industry, medicine, finance, military and other fields are also very broad. However, at the same time, the basic theoretical framework of information fusion technology and effective generalized fusion models and algorithms have not yet been formed. The main direction of current research is the formation of new algorithms and the improvement of existing algorithms, and the integration of these technologies, in the diverse information Integration and application of a unified structure. Reliable membership degree acquisition methods and basic probability replication are the key and technical difficulties for the establishment of the fusion system, and methods and systems for rigorous testing and evaluation of algorithms have not been established. The problem of conversion between theory and application still remains to be solve. New systems and technologies will be further developed under the promotion of interdisciplinary exchanges and research. The focus of future research is still on artificial intelligence and neural network methods. In the two fields of target recognition and robust multi-sensor systems, neural networks Will play an important role.

\section{Basic Concepts and Necessity of Big Data and Cloud Storage}

When a data set reaches a certain size, it can be called "big data." These data may come from different sources, different types, and have many different formats. Big data can be used as the basis for industry data analysis because of its high authenticity and the ability to process data in real time. But as a new type of processing mode, big data analysis cannot be done through traditional tools and processes. Cloud storage is an emerging network storage method, which is based on cloud computing. Through the analysis of network technology and file distributed processing functions and application data collection, cloud storage can integrate various storage devices in the network 
into a whole through corresponding application software, and coordinate the work of various devices. In this way, industry users can store and access data. Big data is the object that cloud computing needs to process. Because the volume of data is too large, the storage capacity of storage devices is very high. Due to technical constraints, the storage capacity of existing physical devices is far from meeting the needs of big data for storage devices, and cloud storage devices that can be infinitely expanded under the cloud computing system have become cloud computing system data storage and data the core of management.

Since cloud storage can reasonably allocate storage space according to the different needs of users, it can also expand the cloud storage space in an unlimited amount, thereby effectively reducing the storage cost of a large amount of data, so compared with other data storage technologies It has the competitive advantage of large storage capacity and low cost. The structure of separation of control and data enables cloud storage to concurrently access huge data, which improves the efficiency of users' access to big data. The big data stored in the cloud is protected by special encryption settings, which effectively improves the security of access. Only specific users with special identities can access it. With the above advantages, cloud storage has become the preferred technology for big data storage in many industries.

\section{Improvement and Innovation of Cloud Storage Technology}

As the use of cloud storage technology becomes more and more popular, users are paying more and more attention to the safety and reliability of data in the storage process. With the advent of the era of big data, the degree of informatization is getting higher and higher, but the security of information and data is getting lower and lower. The leakage of personal information has become the norm in people's daily lives. The security of cloud storage technology Performance and reliability are constantly challenged. Faced with this situation, cloud storage technology should first respond to virus attacks and hacker attacks through professional security software and firewall settings. Then it should also control the user's access procedures, ensure the safety of user information and data in storage, and defend against intrusions and stored bad data, which threatens the security of cloud storage technology. The third is to ensure the confidentiality of cloud storage technology, set up a special storage path for users' business secrets or political secrets with high confidentiality requirements, to meet the needs of users, strictly monitor and manage highly confidential data, To prevent the leakage of data and increase users' trust in cloud storage technology.

The era of big data has come, but the development of cloud storage is still in its infancy. Therefore, it is necessary to design cloud storage specifically at this stage to ensure that the design of cloud storage can satisfy the experience of different groups. Using cloud storage for pure data storage and basic data acquisition only needs to pay for the Internet, no other fees, but if you need to obtain higher quality data or use higher-level storage functions, you need to pay extra. For example, we often use Baidu and Wangyi in our lives and work. Baidu usually provides customers with relatively popular knowledge, which is used very frequently in public reading and data storage and acquisition. Users can perform according to their own needs. Data and information upload, storage and exchange activities. In contrast, the information uploaded by CNKI condenses the wisdom and energy of the author. In order to ensure a good cloud storage order, users need to pay extra for data storage and acquisition. The design of cloud storage needs to be based on the needs of users, and full consideration should be given to the cost of using cloud storage, the size of the data, and the charging standards of companies of different sizes.

The development time of cloud storage technology is still short, and a complete set of industry specifications has not yet been formed. In order to ensure that the development of cloud storage technology can be more healthy, it is necessary to formulate a set of practical and feasible measures in the development and application of cloud storage technology. Implement specifications. Cloud storage technology should first formulate the most basic network specifications to ensure that cloud storage technology can be managed and operated normally under relevant regulations. In addition, corresponding laws and regulations should be formulated to guarantee the security and 
confidentiality of cloud storage data, and to delineate possible rights disputes and infringements. Information and communication technology has developed in a spurt, although Major medical institutions in China are still facing the problem of high infrastructure management costs, but this has prompted medical institutions to introduce a "cloud storage" operation model. For example, surgical videos can provide a new mode of communication between doctors and patients through "cloud storage". The operation model based on "cloud storage" can not only promote communication between doctors, but also ensure the treatment of patients in remote areas and lower-level hospitals. At present, my country's medical industry has gradually begun to use "cloud storage” for information exchange to ensure collaborative development with other original systems and cross-platform compatibility.

\section{Application of Cloud Storage Technology in Big Data Scenarios}

The advent of the era of big data brings challenges to cloud storage technology, but also brings new development opportunities, provides new ideas for its development, and continues to expand its application range. At this stage, the application of big data in Internet operations has attracted more attention. Its penetration in the Internet field has not only greatly improved the efficiency and quality of Internet operations, but also met the different needs of users, which is conducive to improving user satisfaction. Faced with this opportunity, Internet operators must cherish this opportunity, increase their emphasis on cloud storage technology, improve and innovate their own operating methods, integrate cloud technology into it reasonably, and promote their own development to keep pace with the times, In order to obtain considerable development. Cloud storage technology can be used to establish a cloud storage platform, which can not only provide users with richer information, but also break through the limitations of time and space to update relevant information in a timely manner, which has a significant impact on the efficiency of various data resource applications. Significantly, it can promote the efficient operation of the Internet.

With the progress of the times, the improvement of social and economic levels, and the acceleration of the construction of modern cities, people's demand for video surveillance has become stronger. Therefore, the number of installations of video surveillance continues to increase. Its installation can not only provide a reliable basis for dealing with related problems and have a restraining effect on people's behavior, but also meet the development needs of the current society, which is conducive to promoting a civilized and harmonious society. Construction. The reason why video surveillance has good surveillance effects has attracted more and more attention and attention. A large part of the reason is that it incorporates cloud storage technology. Cloud storage technology can effectively manage the monitored data, not only can greatly improve the clarity of the video, but also can store a large amount of monitoring data, which can ensure the integrity of the data, and the storage time is relatively long. In addition, the application of cloud storage technology to video surveillance has also expanded the scope of surveillance to a certain extent, which is conducive to enabling people to obtain more information, and it is helpful to people to carry out distributed management.

In order to meet the needs of work or life, people often need to adjust and reserve resources in the process of storing data or information. The specific adjustment should be based on actual business needs, which may be to increase some storage devices or to reduce some devices. In order to ensure the integrity of the data, during the adjustment, the business cannot be interrupted, nor can it affect other businesses. In this process, if there is deleted data, people must store it in another system in time, and use backup technology to back up and store it.

\section{Conclusion}

my country's big data cloud storage technology based on information fusion technology is still in the initial stage of development, and a lot of theoretical research and more in-depth practical application are needed in the future. As the main feature of the information age, integration is an important direction for the future development of big data cloud storage technology. The advent of 
the big data era has prompted revolutionary changes in all aspects of people's lives and work. Except for the Internet and related industries, in-depth applications in other industries still need a long period of research and running-in, which is relatively lagging. The advent of the era of big data provides new ideas and methods for the management and development of various industries.

\section{References}

[1] Xu Xin. Application Research of Big Data Point Cloud Storage Technology Based on PostgreSQL Database. Information and Computer, no. 13, pp. 21, 2017.

[2] Liu Tiancheng, Cheng Qian, Liu Gao, et al. Research on Information Fusion Technology of Structural Health Monitoring of Pingtang Bridge Based on BIM Platform. Highway, vol. 4, no.9, pp. 18-22, 2019.

[3] Pang Chao, Liu Qian, Wei Hongyu. Research on the big data backup mode of postal training based on cloud storage. Application of Automation, vol. 8, no. 9, pp. 65-67, 2018.

[4] Sun Liuxin. Research on Key Technologies of Big Data Fusion System Based on Cloud Computing. Digital Communication World, vol. 2, no. 1, pp. 56-56, 2016.

[5] Hou Jialu, Hu Xiaofeng, Wang Runhua. Application of Cloud Storage Technology Based on Big Data Environment. Curriculum Education Research: New Teacher Teaching, vol. 12, no. 17, pp. 284-284, 2015. 